\title{
Article \\ Plasma-Generated X-ray Pulses: Betatron Radiation Opportunities at EuPRAXIA@SPARC_LAB
}

\author{
Francesco Stellato ${ }^{1,2, *(D)}$, Maria Pia Anania ${ }^{3}$, Antonella Balerna ${ }^{3}$, Simone Botticelli ${ }^{2}$, Marcello Coreno ${ }^{3,4}$, \\ Gemma Costa $^{3}$, Mario Galletti 1,2, Massimo Ferrario ${ }^{3}$, Augusto Marcelli ${ }^{3,5,6}$, Velia Minicozzi 1,2, Silvia Morante ${ }^{1,2}$,
} Riccardo Pompili ${ }^{3}$, Giancarlo Rossi ${ }^{1,2,7}$, Vladimir Shpakov ${ }^{3}$, Fabio Villa ${ }^{3}$ and Alessandro Cianchi ${ }^{1,2}$

\section{check for}

updates

Citation: Stellato, F.; Anania, M.P.; Balerna, A.; Botticelli, S.; Coreno, M.; Costa, G.; Galletti, M.; Ferrario, M.; Marcelli, A.; Minicozzi, V.; et al. Plasma-Generated X-ray Pulses: Betatron Radiation Opportunities at EuPRAXIA@SPARC_LAB. Condens. Matter 2022, 7, 23. https://doi.org/ $10.3390 /$ condmat7010023

Academic Editors: Bernardo Barbiellini and Atsushi Fujimori

Received: 25 October 2021

Accepted: 21 February 2022

Published: 24 February 2022

Publisher's Note: MDPI stays neutral with regard to jurisdictional claims in published maps and institutional affiliations.

Copyright: (C) 2022 by the authors. Licensee MDPI, Basel, Switzerland. This article is an open access article distributed under the terms and conditions of the Creative Commons Attribution (CC BY) license (https:// creativecommons.org/licenses/by/ $4.0 /)$
1 Physics Department, University of Rome Tor Vergata, Via della Ricerca Scientifica 1, 00133 Roma, Italy; mario.galletti@lnf.infn.it (M.G.); minicozzi@roma2.infn.it (V.M.); morante@roma2.infn.it (S.M.); rossig@roma2.infn.it (G.R.); alessandro.cianchi@uniroma2.it (A.C.)

2 INFN_Sezione di Roma Tor Vergata, Via della Ricerca Scientifica 1, 00133 Roma, Italy; botticelli@roma2.infn.it

3 INFN_Laboratori Nazionali di Frascati, Via Enrico Fermi 54, 00044 Frascati, Italy; maria.pia.anania@lnf.infn.it (M.P.A.); balerna@lnf.infn.it (A.B.); marcello.coreno@elettra.eu (M.C.); gemma.costa@lnf.infn.it (G.C.); massimo.ferrario@lnf.infn.it (M.F.); augusto.marcelli@lnf.infn.it (A.M.); riccardo.pompili@lnf.infn.it (R.P.); vladimir.shpakov@lnf.infn.it (V.S.); fvilla@lnf.infn.it (F.V.)

4 Istituto Struttura della Materia, C.N.R., LD2 Unit, 34149 Trieste, Italy

5 Istituto Struttura della Materia, C.N.R., Via del Fosso del Cavaliere 100, 00133 Rome, Italy

6 RICMASS, Rome International Center for Materials Science Superstripes, 00185 Rome, Italy

7 Centro Fermi-Museo Storico della Fisica e Centro Studi e Ricerche E. Fermi, 00184 Roma, Italy

* Correspondence: stellato@roma2.infn.it

\begin{abstract}
EuPRAXIA is a leading European project aimed at the development of a dedicated, groundbreaking, ultra-compact accelerator research infrastructure based on novel plasma acceleration concepts and laser technology and on the development of their users' communities. Within this framework, the Laboratori Nazionali di Frascati (LNF, INFN) will be equipped with a unique combination of an X-band RF LINAC generating high-brightness GeV-range electron beams, a $0.5 \mathrm{PW}$ class laser system and the first fifth-generation free electron laser (FEL) source driven by a plasma-based accelerator, the EuPRAXIA@SPARC_LAB facility. Wiggler-like radiation emitted by electrons accelerated in plasma wakefields gives rise to brilliant, ultra-short X-ray pulses, called betatron radiation. Extensive studies have been performed at the FLAME laser facility at LNF, INFN, where betatron radiation was measured and characterized. The purpose of this paper is to describe the betatron spectrum emitted by particle wakefield acceleration at EuPRAXIA@SPARC_LAB and provide an overview of the foreseen applications of this specific source, thus helping to establish a future user community interested in (possibly coupled) FEL and betatron radiation experiments. In order to provide a quantitative estimate of the expected betatron spectrum and therefore to present suitable applications, we performed simple simulations to determine the spectrum of the betatron radiation emitted at EuPRAXIA@SPARC_LAB. With reference to experiments performed exploiting similar betatron sources, we highlight the opportunities offered by its brilliant femtosecond pulses for ultra-fast X-ray spectroscopy and imaging measurements, but also as an ancillary tool for designing and testing FEL instrumentation and experiments.
\end{abstract}

Keywords: free electron lasers; betatron emission; X-ray spectroscopy; phase-contrast imaging

\section{Introduction}

EuPRAXIA is a European project aimed at developing a particle accelerator research infrastructure based on plasma acceleration [1]. The EuPRAXIA@SPARC_LAB facility will provide the Laboratori Nazionali di Frascati (LNF) with a unique combination of a high-brightness GeV-range electron beam accelerated by a state-of-the-art X-band RF linac, a 0.5 PW class laser system and an ultimate light source driven by a plasma accelerator [2]. The EuPRAXIA@SPARC_LAB scientific program includes, among its main 
research directions, the development of advanced radiation sources for photon science. In plasma acceleration, a driver pulse, which can be either a laser or an electron (or, in general, charged particles) beam, creates a wakefield in a plasma, referred to as Laser Wakefield Accelerator (LWFA) and Particle Wakefield Accelerator (PWFA), respectively. The plasma wakefield accelerates a witness electron bunch injected behind the driver pulse. The electrons wiggle in the plasma wakefield and are accelerated at a few $\mathrm{GeV} / \mathrm{m}$. The accelerated electrons are then injected into an undulator and generate FEL pulses. At EuPRAXIA@SPARC_LAB, the FEL photons will have a wavelength of approximately $3 \mathrm{~nm}$, therefore falling within the energy region that lies between the carbon and oxygen K-edge, the so-called water window. The foreseen applications of this FEL radiation have been discussed elsewhere [3]. However, the FEL beam is not the only photon source that can be obtained from plasma acceleration. It is worth noting that electrons moving on an oscillatory trajectory at relativistic velocity, as happens in LWFA or PWFA, emit photons. The characteristics of this radiation, which is referred to as betatron radiation, depend on the electron beam parameters [4,5]. Betatron radiation with a certain degree of coherence is emitted within a narrow cone in a process analogous to wiggler emission in storage ring light sources. The first measurements of betatron radiation emitted in laser-plasma acceleration experiments at the LNF were performed at the FLAME (Frascati Laser for Acceleration and Multidisciplinary Experiments) laser facility [6,7]. The measured betatron radiation spectrum was in the X-ray region from $2 \mathrm{keV}$ to $25 \mathrm{keV}$. As detailed in the next sections, also at the EuPRAXIA@SPARC_LAB parameters, the emitted betatron radiation is expected to lie in the $\mathrm{X}$-ray region. Betatron sources share the common properties of being compact and delivering collimated, femtosecond radiation [8] and have a broad range of applications, including biomedical applications, such as radiology; defense and industrial applications, including the detection of explosives and drugs; and high-energy-density science, aimed at the study of fusion plasmas and planetary and stellar interiors [9]. In the field of condensed matter physics, these sources are particularly useful when a short pulse length is required. In this paper, we report preliminary simulation data about the betatron radiation properties at EuPRAXIA@SPARC_LAB and we describe some possible applications compatible with the simulated spectrum parameters.

\section{Results}

\subsection{Simulations}

Simulations to estimate the intensity and energy spectrum of the betatron radiation have been performed starting from the EuPRAXIA@SPARC_LAB design parameters, as detailed in Table 1. The linac will be capable of providing energy up to $600 \mathrm{MeV}$, which will be increased to $950 \mathrm{MeV}$ thanks to the plasma module. Regarding the capillary length, at present, we have already reached the discharge in $20-\mathrm{cm}-$ long capillaries. For the EuPRAXIA@SPARC_LAB design, we are working on sapphire capillaries that should be able to reach the indicated length. A possibility that we are exploring is to work with a segmented capillary, consisting of three $20 \mathrm{~cm}$ capillaries placed all together, without spacing in between.

Table 1. EuPRAXIA@SPARC_LAB parameters.

\begin{tabular}{cc}
\hline Parameter [Units] & Value \\
\hline Beam energy [MeV] & $600-950$ \\
Plasma density [cm $\left.{ }^{-3}\right]$ & $3.0 \times 10^{16}$ \\
RMS transverse beam size $[\mu \mathrm{m}]$ & 3.0 \\
Charge [pC] & 45 \\
Capillary (plasma) length $[\mathrm{m}]$ & 0.6 \\
Pulse duration [fs] & 10 \\
\hline
\end{tabular}


The betatron radiation spectrum arising from the plasma acceleration was calculated following the models provided in Refs. [10,11], where it is assumed that the electrons in the plasma are oscillating only in one plane. In more detail, in these models, the plasma is considered as a simple ion column or focusing channel. The electrons are oscillating inside it with a plane sinusoidal trajectory. The parameters of this trajectory are therefore defined by the beam parameters (transverse size, energy) and plasma density. Since the models work for a constant energy, in order to take into account acceleration, we divide the capillary into several sections and calculate the betatron radiation coming from these sections, increasing the energy from section to section, while keeping the energy constant on each section. The expression that we use for the betatron radiation on each section comes from Equation (30) in Ref. [11]. Figure 1 shows the betatron spectra simulated for three different plasma densities. According to these simulations, the betatron spectrum will range from around $1 \mathrm{~nm}$ down to the hard X-ray region (less than $0.1 \mathrm{~nm}$ ). The blue curve is obtained for a plasma density of $3.0 \times 10^{16} \mathrm{~cm}^{-3}$, which corresponds to the EuPRAXIA@SPARC_LAB baseline operation value. Since it is possible to increase the photon flux by increasing the plasma density, we compare the "baseline" spectrum with those simulated assuming higher plasma densities. The scenarios that we outline here are still compatible with the experimental design and can be used as reliable models to maximize the betatron emission. The critical energy is, approximately, $5 \mathrm{keV}, 3.5 \mathrm{keV}$ and $1.5 \mathrm{keV}$ for plasma densities of $9.0 \times 10^{16} \mathrm{~cm}^{-3}, 6.0 \times 10^{16} \mathrm{~cm}^{-3}$ and $3.0 \times 10^{16} \mathrm{~cm}^{-3}$, respectively. However, it is worth pointing out that, although the spectrum resembles bending magnet synchrotron radiation, it actually corresponds to the wiggler emission in storage ring light sources, with a series of maxima (harmonics), which, due to the fact that the deflection angle of the oscillatory motion is high, simply merge into what becomes a continuous spectrum.

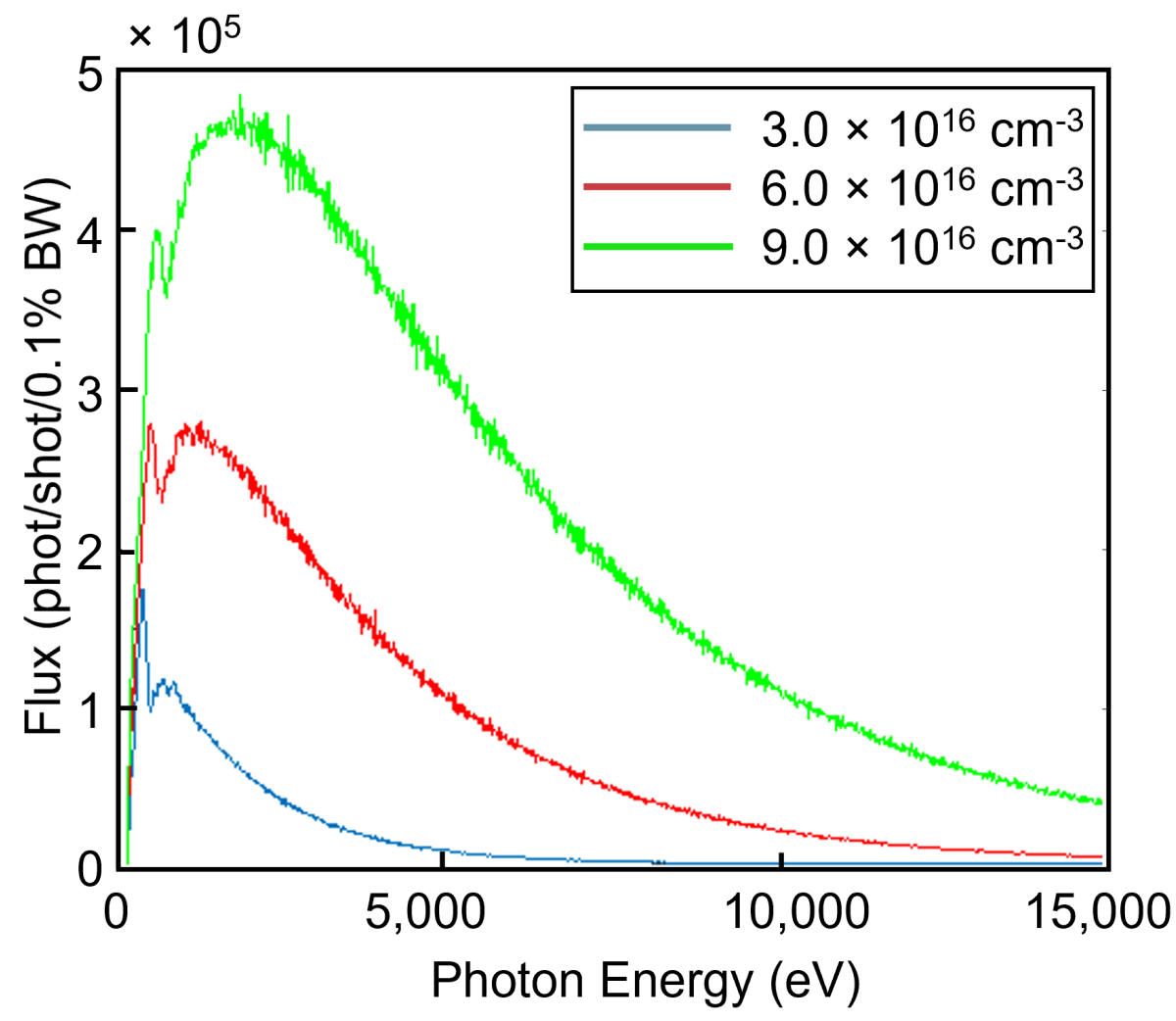

Figure 1. Betatron radiation spectra simulated for a source size of $3 \mu \mathrm{m}$ and 3 different plasma densities. The total number of photons is $1.7 \times 10^{9}$ for the $9.0 \times 10^{16} \mathrm{~cm}^{-3}$ density, $9.9 \times 10^{8}$ for the $6.0 \times 10^{16} \mathrm{~cm}^{-3}$ density and $4.1 \times 10^{8}$ for the $3.0 \times 10^{16} \mathrm{~cm}^{-3}$ density. 


\subsection{Betatron Imaging}

In "traditional" imaging (and computer tomography, CT), one measures the variation in the X-ray absorption coefficient in different parts of a sample. In phase-contrast imaging (PCI), one measures instead the difference in wavefront. In this way, i.e., by making the phase gradient visible, PCI makes it possible to visualize soft-tissue structures that are otherwise unresolved with CT. This method thus improves the resolution in soft tissue imaging (e.g., lungs and brain) and typically requires lower doses [12]. PCI measurements require an $\mathrm{X}$-ray radiation source. Betatron-based sources could fill the gap between synchrotron- and X-ray tube-based sources in terms of photon flux and the size of the photon source $[13,14]$, providing a novel tool to perform PCI but also CT measurements [15]. The application of bright, ultrafast $\mathrm{X}$-ray radiation to the phase-contrast imaging of biological specimens was demonstrated in 2011 in [16].

In [13], Guo and coworkers showed that good-quality PCI images can be obtained within $1 \mathrm{~min}$ at a repetition rate of $0.3 \mathrm{~Hz}$, with approximately $1.1 \times 10^{7}$ photons per shot in the energy range between 4 and $15 \mathrm{keV}$. Considering that EuPRAXIA@SPARC_LAB will have, according to the simulations shown in Figure 1, approximately $4 \times 10^{8}$ photons per shot in the 4-15 keV energy range (for the $6.0 \times 10^{16} \mathrm{~cm}^{-3}$ density case), and a target repetition rate of $10 \mathrm{~Hz}$, images of quality comparable with Ref. [13] can be acquired on even shorter timescales, provided that the source will exhibit good stability.

Simulations suggest that a photon flux of order $10^{9}$ photons/shot might even allow us to achieve a sub-micron resolution [17]. Although a sub-micron resolution will not be reached due to the limited photon flux, EuPRAXIA@SPARC_LAB will still provide betatron photons to perform PCI measurements on biological specimens, such as insects or tissues. This will favor the broader adoption of advanced imaging techniques, such as PCI, in university-sized laboratories [18], thus paving the way for the development of small-scale sources for PCI.

\subsection{Betatron X-ray Spectroscopy}

As with wigglers, betatron sources have a broad energy spectrum, but they emit very short pulses. They are therefore ideal sources to perform time-resolved spectroscopic measurements, such as X-ray absorption spectroscopy (XAS).

XAS is indeed a powerful technique to study the electronic and atomic structures around selected absorbing atoms. In addition, time-resolved XAS experiments can therefore provide information on the dynamics induced in a sample by an external pump. In order to perform these experiments with a high temporal resolution, an X-ray source with femtosecond pulses is needed. The first option is to exploit X-ray FEL radiation [19-22]. However, the beamtime demand at FEL sources is larger than the availability. For this reason, at least for the kinds of experiments not requiring the peak brilliance of FELs, other X-ray sources with ultra-short pulses could be used. Among these, betatron radiation sources have the advantages of providing a continuous energy spectrum, ideal to perform spectroscopic experiments, and of having pulses down to the femtosecond domain, ideal to obtain a high time resolution. Betatron experiments have indeed allowed researchers to successfully study the nonequilibrium dynamics of copper at extreme temperatures and pressures [23]. In this experiment, a femtosecond optical laser pulse "pumped" the sample, while a subsequent femtosecond X-ray betatron pulse, with approximately $10^{5}$ photons $/$ shot $/ 0.1 \%$ bandwidth around $1 \mathrm{keV}$, "probed" its dynamics by XAS. Similarly, Mo et al. [24] performed laser-pump, betatron-probe XAS experiments to measure the ionization states in warm dense aluminum.

In order to assess the feasibility of XAS experiments exploiting the betatron source described in the present paper, we observe that, in Ref. [23], the experiments were performed with a beam providing $10^{5}$ photons $/$ shot $/ 0.1 \%$ bandwidth [8]. A total of 50 acquisitions (at $0.3 \mathrm{~Hz}$ ) were merged to obtain the spectra analyzed in the paper. According to the simulations shown in Figure 1, this number is well in the reach of the EuPRAXIA@SPARC_LAB betatron emission, which can be as high as $5 \times 10^{5}$ photons/shot in the most optimistic 
scenario, with the highest plasma density, but will be sufficient also in the less optimistic one. Considering the higher repetition rate of EuPRAXIA@SPARC_LAB, it is foreseeable that it will allow data collection on similar samples in shorter times.

It is also possible to perform laser pump-X-ray probe experiments by using the same laser pulse to pump the system and generate the betatron radiation. In [25], the optical system splits a laser beam into two beams, one serving as a pump to excite $\mathrm{SiO}_{2}$, while the second one produces the betatron radiation in a laser wakefield accelerator. The experiment, performed at the Matter under Extreme Conditions end-station of the Linac Coherent Light Source (LCLS), allowed the production of laser and X-ray pulses with adjustable delays and the acquisition of time-resolved XAS data. Following these works, it is expected that the EuPRAXIA@SPARC_LAB betatron radiation will be suitable to perform XAS experiments. The expected brilliance is lower than that which can be achieved at FELs; therefore, its application will be likely limited to highly absorbing samples. It is worth pointing out that this still leaves space for the investigation of phenomena in which time resolution is crucial. Laser pump-X-ray probe schemes will be possible also at EuPRAXIA@SPARC_LAB, although, in the presented configuration, the betatron radiation is emitted by PWFA, and so the timing between pump and probe pulses will not be automatically set.

Precise determination of the X-ray pulse arrival time is necessary for accurate analysis of pump-probe experiments as it allows one to compensate for the temporal jitter of the photon pulses. Streaking techniques, in which the X-ray pulse is overlapped with overlapping an IR or THz in a gas, are routinely used for this purpose. Ref. [26], although other methods are currently used at FEL sources, based on ultrafast free-carrier generation in optically transparent materials, might also be adapted [27].

\subsection{Betatron Test Chamber}

X-ray experiments are typically performed in vacuum chambers hosting the sample holder, one or more detectors, the optics and the diagnostics and the motorized stages to align all these elements. The setup of the experimental chamber is particularly important for FEL experiments, where the radiation has a pulsed time structure and, due to its high brilliance, its interaction with the samples can be destructive. Moreover, given the need for maximizing the actual data acquisition rate during an FEL beamtime, alignment is a crucial step. Concerning the samples, different delivery systems may be used, each one requiring care not only regarding the mechanical alignment of the samples, but also, when dealing with liquid jets or aerosol sources, in terms of operating pressures and of the interaction of the sample itself and of the gases or liquids used for its injection with the radiation. For these reasons, test chambers, able to host a sample delivery setup identical to the one described, are widely used at FELs. At the European XFEL, a vacuum chamber hosting a gas-dynamic virtual nozzle injection system is available for the users to optimize the working conditions before the experiments [28].

At the LCLS, an FEL simulator, an endstation that mimics the LCLS experimental sample environment, is also available. It consists of a vacuum chamber coupled to multiple laser sources and able to accommodate various sample delivery tools. A laser is focused in the center of the chamber to irradiate the sample. Diagnostics include a time-of-flight detector for analyzing the remnants of the photoionized sample, as well as high-speed cameras and optical detectors to collect scattered photons and images of jet breakup. One key point of these test chambers is that they are equipped with a photon source able to emulate the FEL radiation. In this respect, femtosecond optical lasers are relatively cheap and easy to use and can offer an FEL-like time structure. However, their wavelength is much larger than that of FEL sources. A betatron source would have the advantage of providing X-ray femtosecond photon pulses. In the specific case of EuPRAXIA@SPARC_LAB, these pulses would naturally have the same repetition rate and duration of the FEL pulses. For this reason, at EuPRAXIA@SPARC_LAB, a vacuum chamber, fed by the betatron radiation, will be installed. This test facility will be able to serve as a test chamber for FEL 
experiments performed at EuPRAXIA@SPARC_LAB itself and, possibly, also at other FEL radiation sources.

A sketch of the test chamber foreseen for EuPRAXIA@SPARC_LAB, including various sample delivery systems and photons and ion detectors, is provided in Figure 2, which provides a schematic view of a setup for X-ray experiments at the EuPRAXIA@SPARC_LAB facility.

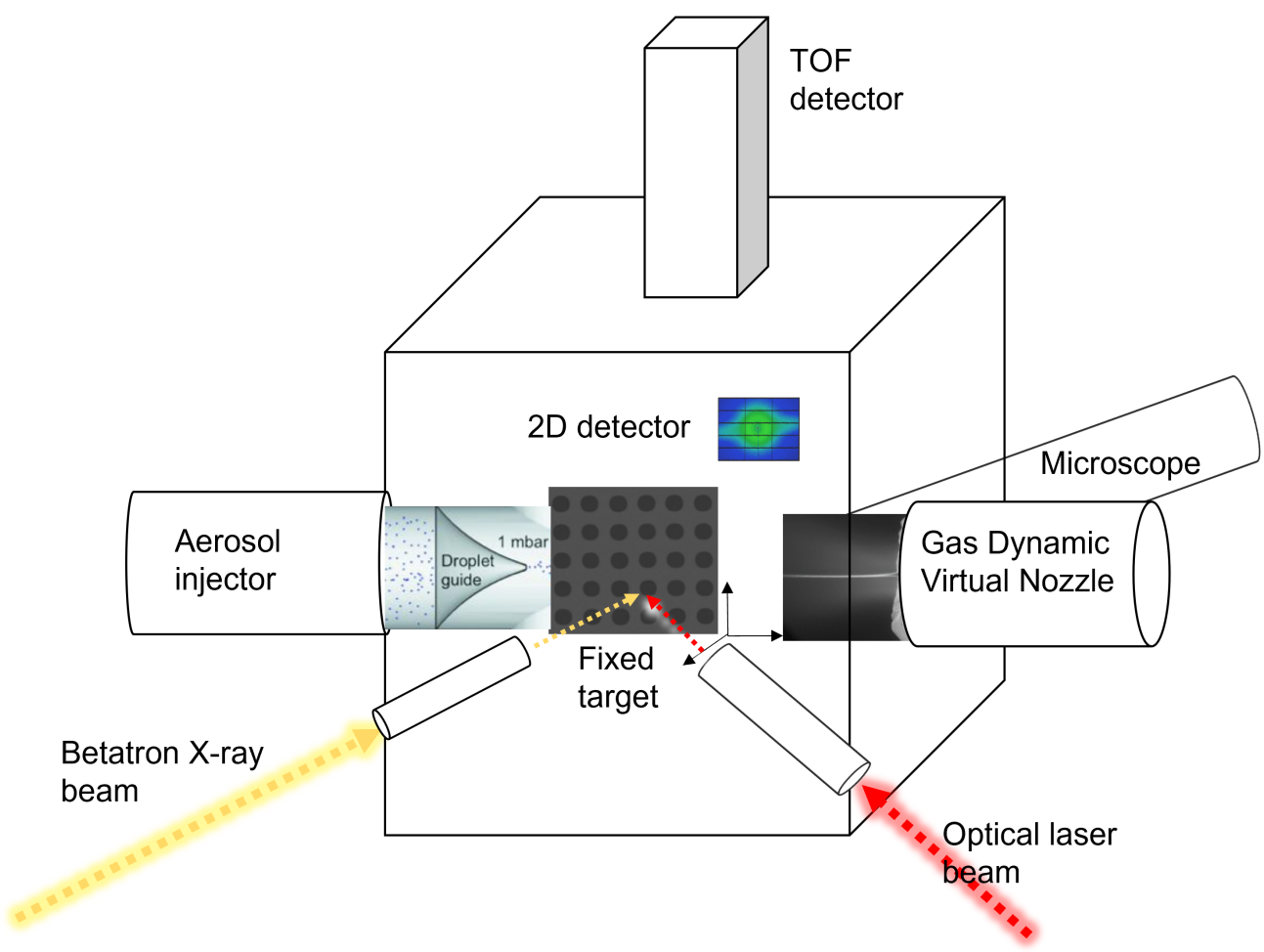

Figure 2. A schematic view of the test chamber to be installed at the EuPRAXIA@SPARC_LAB facility.

\section{Discussion}

As discussed above, betatron radiation can be considered a by-product of a plasma accelerator. Within a circular economy framework, this is a good reason to exploit this facility as its best. Given the framework of EuPRAXIA@SPARC_LAB as a laboratory to produce and exploit plasma acceleration and to serve as a photon source, it is perfectly in line with the scope of this project to foresee and discuss the possible application of betatron radiation. In this context, the first simulations show that pulses with a duration of a few femtoseconds and more than $10^{9} \mathrm{X}$-ray photons may be produced, with a brilliance of more than $10^{5}$ photon pulses within a $0.1 \%$ bandwidth. Although the foreseen average brilliance of the EuPRAXIA@SPARC_LAB betatron source is lower than that of synchrotron X-ray sources, the time structure of the radiation, which is characterized by femtosecond pulses, still leads to a very high peak brilliance and allows performing spectroscopy experiments with high time resolution. The coherence properties and the flux will also be suitable for PCI experiments on samples of biomedical interest. Figure 3 provides a schematic view of a setup for X-ray experiments at the EuPRAXIA@SPARC_LAB facility.

Moreover, betatron photons can be used to feed an experimental test chamber to set up experiments to be performed at EuPRAXIA@SPARC_LAB itself, but also at other FEL sources. Finally, since the betatron source will be naturally synchronized with the plasmafed FEL, it will be possible to exploit it to perform time-resolved betatron pump/FEL probe (or FEL pump/X-ray probe) experiments. In summary, it is worth pointing out that, although the EuPRAXIA@SPARC_LAB betatron source will not necessarily be brighter than other existing betatron sources, it will exhibit the interesting feature of being able 
to operate in conjunction with an FEL source. This setting will offer the possibility of performing not only stand-alone experiments but also complementary measurements, such as PCI and spectroscopy, as well as serving as a support for the FEL experiments themselves. In this context, the femtosecond betatron X-ray pulses can be exploited to feed a test chamber in test runs for FEL experiments. This will have the advantage, compared to femtosecond lasers used in similar situations, of providing the user with a much shorter photon wavelength. Moreover, at this project-definition stage, it is still possible for the future user community to come up with specific ideas that can be implemented in the design of the betatron source and of the related instrumentation.

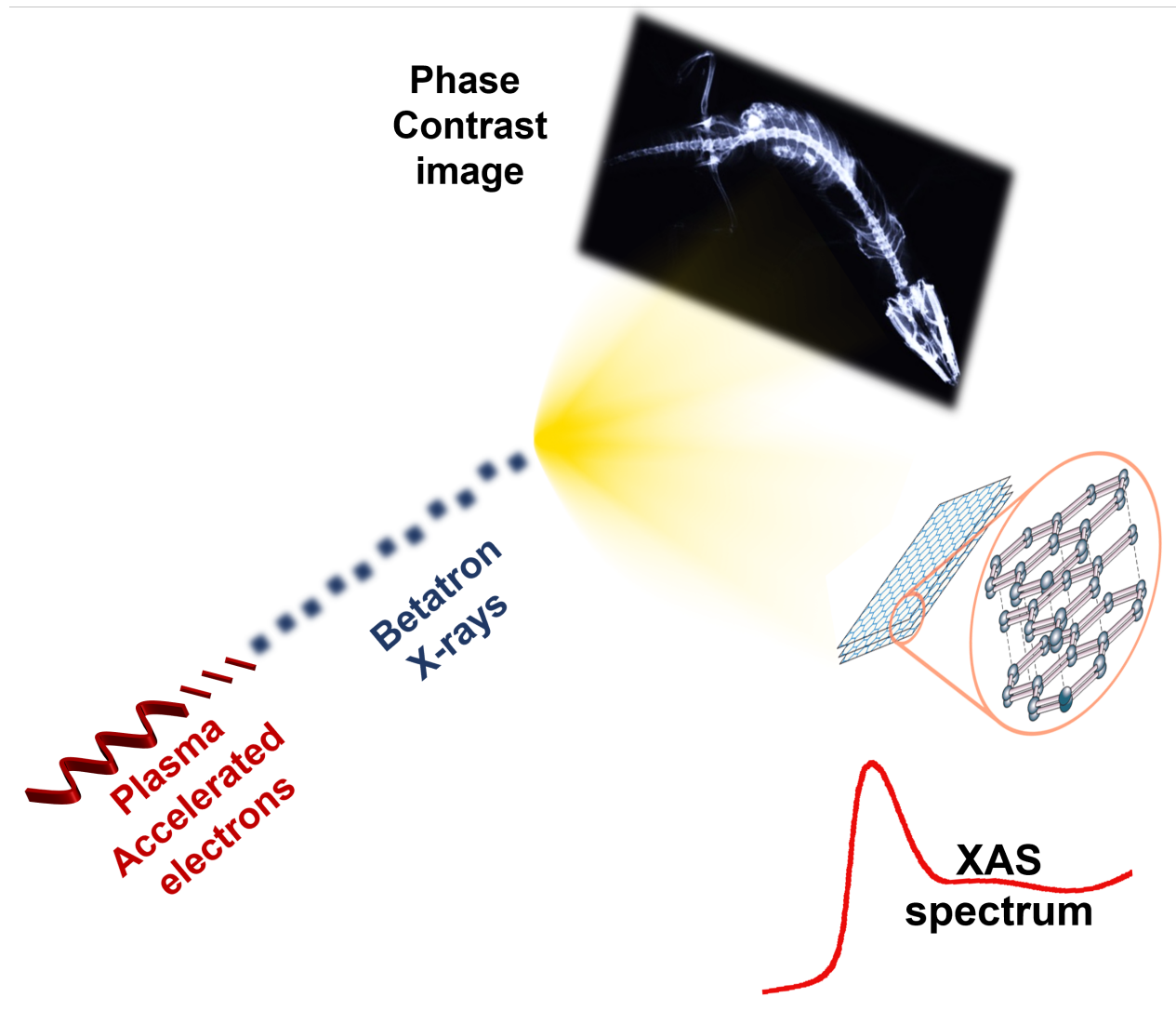

Figure 3. A schematic view of PCI and XAS experiments at the EuPRAXIA@SPARC_LAB facility. The PCI image of the lacewing insect (chrysoperia carnea) has been adapted from [14].

Author Contributions: Conceptualization, F.S. and V.S.; Funding acquisition, M.F.; Investigation, V.S.; Methodology, F.S., M.P.A., A.B., G.C., M.G., M.F., A.M., R.P., V.S., F.V. and A.C.; Project administration, M.F.; Visualization, S.B.; Writing—original draft, F.S., M.G., M.F. and A.C.; Writing-review \& editing, M.P.A., A.B., M.C., G.C., A.M., V.M., S.M., G.R. and F.V. All authors have read and agreed to the published version of the manuscript.

Funding: This work was supported by the European Union's Horizon 2020 research and innovation program under grant agreement No. 653782 (EuPRAXIA).

Institutional Review Board Statement: Not applicable.

Informed Consent Statement: Not applicable.

Data Availability Statement: The data that support the simulations of this study are available from the corresponding author upon reasonable request.

Conflicts of Interest: The authors declare no conflict of interest. 


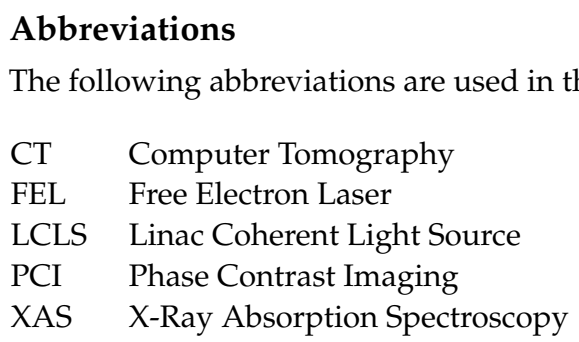

\section{References}

1. Assmann, R.; Weikum, M.; Akhter, T.; Alesini, D.; Alexandrova, A.; Anania, M.; Andreev, N.; Andriyash, I.; Artioli, M.; Aschikhin, A.; et al. EuPRAXIA conceptual design report. Eur. Phys. J. Spec. Top. 2020, 229, 3675-4284. [CrossRef]

2. Ferrario, M.; Alesini, D.; Anania, M.; Artioli, M.; Bacci, A.; Bartocci, S.; Bedogni, R.; Bellaveglia, M.; Biagioni, A.; Bisesto, F.; et al. EuPRAXIA@SPARC_LAB Design study towards a compact FEL facility at LNF. Nucl. Instrum. Methods Phys. Res. Sect. A Accel. Spectrometers Detect. Assoc. Equip. 2018, 909, 134-138. [CrossRef]

3. Balerna, A.; Bartocci, S.; Batignani, G.; Cianchi, A.; Chiadroni, E.; Coreno, M.; Cricenti, A.; Dabagov, S.; Di Cicco, A.; Faiferri, M.; et al. The Potential of EuPRAXIA@ SPARC_LAB for Radiation Based Techniques. Condens. Matter 2019, 4, 30. [CrossRef]

4. Paroli, B.; Chiadroni, E.; Ferrario, M.; Mostacci, A.; Petrillo, V.; Potenza, M.; Rossi, A.; Serafini, L. Coherence properties and diagnostics of betatron radiation emitted by an externally-injected electron beam propagating in a plasma channel. Nucl. Instrum. Methods Phys. Res. Sect. B Beam Interact. Mater. Atoms 2015, 355, 217-220. [CrossRef]

5. Guo, B.; Xu, X.; Hua, J.; Wu, Y.; Pai, C.H.; Lu, W. Generation of Coherent Monochromatic Betatron Radiation by Laser-triggered Ionization Injection in Plasma Accelerators. In Proceedings of the 2018 IEEE Advanced Accelerator Concepts Workshop (AAC), Breckenridge, CO, USA. 12-17 August 2018; pp. 1-4.

6. Curcio, A.; Anania, M.; Bisesto, F.; Chiadroni, E.; Cianchi, A.; Ferrario, M.; Filippi, F.; Giulietti, D.; Marocchino, A.; Mira, F.; et al. First measurements of betatron radiation at FLAME laser facility. Nucl. Instrum. Methods Phys. Res. Sect. B Beam Interact. Mater. Atoms 2017, 402, 388-392. [CrossRef]

7. Curcio, A.; Anania, M.; Bisesto, F.; Chiadroni, E.; Cianchi, A.; Ferrario, M.; Filippi, F.; Giulietti, D.; Marocchino, A.; Petrarca, M.; et al. Trace-space reconstruction of low-emittance electron beams through betatron radiation in laser-plasma accelerators. Phys. Rev. Accel. Beams 2017, 20, 012801. [CrossRef]

8. Corde, S.; Phuoc, K.T.; Lambert, G.; Fitour, R.; Malka, V.; Rousse, A.; Beck, A.; Lefebvre, E. Femtosecond x rays from laser-plasma accelerators. Rev. Mod. Phys. 2013, 85, 1. [CrossRef]

9. Albert, F.; Thomas, A.G. Applications of laser wakefield accelerator-based light sources. Plasma Phys. Control. Fusion 2016, 58,103001 . [CrossRef]

10. Esarey, E.; Shadwick, B.; Catravas, P.; Leemans, W. Synchrotron radiation from electron beams in plasma-focusing channels. Phys. Rev. E 2002, 65, 056505. [CrossRef]

11. Leemans, W.P.; Esarey, E.; van Tilborg, J.; Michel, P.A.; Schroeder, C.B.; Tóth, C.; Geddes, C.G.; Shadwick, B.A. Radiation from laser accelerated electron bunches: Coherent terahertz and femtosecond X-rays. IEEE Trans. Plasma Sci. 2005, 33, 8-22. [CrossRef]

12. Croton, L.C.; Morgan, K.S.; Paganin, D.M.; Kerr, L.T.; Wallace, M.J.; Crossley, K.J.; Miller, S.L.; Yagi, N.; Uesugi, K.; Hooper, S.B.; et al. In situ phase contrast X-ray brain CT. Sci. Rep. 2018, 8, 11412. [CrossRef] [PubMed]

13. Guo, B.; Zhang, X.; Zhang, J.; Hua, J.; Pai, C.H.; Zhang, C.; Chu, H.H.; Mori, W.; Joshi, C.; Wang, J.; et al. High-resolution phase-contrast imaging of biological specimens using a stable betatron X-ray source in the multiple-exposure mode. Sci. Rep. 2019, 9, 7796. [CrossRef] [PubMed]

14. Wenz, J.; Schleede, S.; Khrennikov, K.; Bech, M.; Thibault, P.; Heigoldt, M.; Pfeiffer, F.; Karsch, S. Quantitative X-ray phase-contrast microtomography from a compact laser-driven betatron source. Nat. Commun. 2015, 6, 7568. [CrossRef] [PubMed]

15. Cole, J.M.; Symes, D.R.; Lopes, N.C.; Wood, J.C.; Poder, K.; Alatabi, S.; Botchway, S.W.; Foster, P.S.; Gratton, S.; Johnson, S.; et al. High-resolution $\mu \mathrm{CT}$ of a mouse embryo using a compact laser-driven X-ray betatron source. Proc. Natl. Acad. Sci. USA 2018, 115, 6335-6340. [CrossRef]

16. Kneip, S.; McGuffey, C.; Dollar, F.; Bloom, M.; Chvykov, V.; Kalintchenko, G.; Krushelnick, K.; Maksimchuk, A.; Mangles, S.; Matsuoka, T.; et al. X-ray phase contrast imaging of biological specimens with femtosecond pulses of betatron radiation from a compact laser plasma wakefield accelerator. Appl. Phys. Lett. 2011, 99, 093701. [CrossRef]

17. van Tilborg, J.; Ostermayr, T.; Tsai, H.E.; Schenkel, T.; Geddes, C.; Schroeder, C.; Esarey, E. Phase-contrast imaging with laser-plasma-accelerator betatron sources. In Proceedings of the International Conference on X-ray Lasers 2020, Rome, Italy, 6-11 September 2020; Volume 11886, p. 118860Q.

18. Najmudin, Z.; Kneip, S.; Bloom, M.; Mangles, S.; Chekhlov, O.; Dangor, A.; Döpp, A.; Ertel, K.; Hawkes, S.; Holloway, J.; et al. Compact laser accelerators for X-ray phase-contrast imaging. Philos. Trans. R. Soc. A Math. Phys. Eng. Sci. 2014, 372, 20130032. [CrossRef]

19. Brenner, G.; Dziarzhytski, S.; Miedema, P.S.; Rösner, B.; David, C.; Beye, M. Normalized single-shot X-ray absorption spectroscopy at a free-electron laser. Opt. Lett. 2019, 44, 2157-2160. [CrossRef] 
20. Kroll, T.; Kern, J.; Kubin, M.; Ratner, D.; Gul, S.; Fuller, F.D.; Löchel, H.; Krzywinski, J.; Lutman, A.; Ding, Y.; et al. X-ray absorption spectroscopy using a self-seeded soft X-ray free-electron laser. Opt. Express 2016, 24, 22469-22480. [CrossRef]

21. Mitzner, R.; Rehanek, J.; Kern, J.; Gul, S.; Hattne, J.; Taguchi, T.; Alonso-Mori, R.; Tran, R.; Weniger, C.; öder, H.; et al. L-edge x-ray absorption spectroscopy of dilute systems relevant to metalloproteins using an x-ray free-electron laser. J. Phys. Chem. Lett. 2013, 4, 3641-3647. [CrossRef]

22. Levantino, M.; Lemke, H.; Schirò, G.; Glownia, M.; Cupane, A.; Cammarata, M. Observing heme doming in myoglobin with femtosecond X-ray absorption spectroscopy. Struct. Dyn. 2015, 2, 041713. [CrossRef]

23. Mahieu, B.; Jourdain, N.; Phuoc, K.T.; Dorchies, F.; Goddet, J.P.; Lifschitz, A.; Renaudin, P.; Lecherbourg, L. Probing warm dense matter using femtosecond x-ray absorption spectroscopy with a laser-produced betatron source. Nat. Commun. 2018, 9, 3276. [CrossRef] [PubMed]

24. Mo, M.; Chen, Z.; Fourmaux, S.; Saraf, A.; Kerr, S.; Otani, K.; Masoud, R.; Kieffer, J.C.; Tsui, Y.; Ng, A.; et al. Measurements of ionization states in warm dense aluminum with betatron radiation. Phys. Rev. E 2017, 95, 053208. [CrossRef] [PubMed]

25. Albert, F.; Barbrel, B.; Condamine, F.; Dorchies, F.; Falcone, R.; Fernandez, A.; Fletcher, L.; Fry, A.; Galtier, E.; Gamboa, E.; et al. Time-resolved X-ray absorption spectroscopy of warm dense matter with betatron X-ray radiation. In Proceedings of the APS Division of Plasma Physics Meeting Abstracts, Portland, OR, USA, 5-9 November 2018; Volume 2018, p. JO8-003.

26. Juranić, P.; Stepanov, A.; Ischebeck, R.; Schlott, V.; Pradervand, C.; Patthey, L.; Radović, M.; Gorgisyan, I.; Rivkin, L.; Hauri, C.; et al. High-precision x-ray FEL pulse arrival time measurements at SACLA by a THz streak camera with Xe clusters. Opt. Express 2014, 22, 30004-30012. [CrossRef]

27. Harmand, M.; Coffee, R.; Bionta, M.R.; Chollet, M.; French, D.; Zhu, D.; Fritz, D.; Lemke, H.; Medvedev, N.; Ziaja, B.; et al. Achieving few-femtosecond time-sorting at hard X-ray free-electron lasers. Nat. Photonics 2013, 7, 215-218. [CrossRef]

28. Han, H.; Round, E.; Schubert, R.; Gül, Y.; Makroczyová, J.; Meza, D.; Heuser, P.; Aepfelbacher, M.; Barák, I.; Betzel, C.; et al. The XBI BioLab for life science experiments at the European XFEL. J. Appl. Crystallogr. 2021, 54, 7-21. [CrossRef] [PubMed] 